\title{
Cusp-latitude Pc3 spectra: band-limited and power-law components
}

\author{
P. V. Ponomarenko ${ }^{1}$, B. J. Fraser ${ }^{1}$, F. W. Menk ${ }^{1}$, S. T. Ables ${ }^{1, *}$, and R. J. Morris ${ }^{2}$ \\ ${ }^{1}$ University of Newcastle, Callaghan, NSW, Australia \\ ${ }^{2}$ Australian Antarctic Division, Kingston, TAS, Australia \\ *also in: CRC for Satellite Systems, Australia
}

Received: 29 November 2001 - Revised: 26 April 2002 - Accepted: 14 May 2002

\begin{abstract}
This work attempts to fill a gap in comparative studies of upstream-generated Pc3-4 waves and broad band ULF noise observed at cusp latitudes. We performed a statistical analysis of the spectral properties of three years of cusp-latitude ground magnetometer data, finding that the average daytime Pc3-4 spectra are characterized by two principal components: an upstream-related band-limited enhancement ('signal') and a power-law background ('noise') with $S(f) \propto f^{-4}$. Based on this information we developed an algorithm allowing for the deconvolution of these two components in the spectral domain. The frequency of the signal enhancement increases linearly with IMF magnitude as $f[\mathrm{mHz}] \simeq 4.4\left|B_{\mathrm{IMF}}\right|[\mathrm{nT}]$, and its power maximizes around IMF cone angles $\theta_{x B} \simeq 20$ and $160^{\circ}$ and at 10:30-11:00 MLT. Both spectral components exhibit similar semiannual variations with equinoctial maxima. The background noise power grows with increasing southward $B_{z}$ and remains nearly constant for northward $B_{z}$. Its diurnal variation resembles that of Pc5 field-line resonance power, with a maximum near 09:00 MLT. Both the band-limited signal and broad band noise components show power-law growth with solar wind velocity $\propto V_{s w}^{5.71}$ and $\propto V_{s w}^{4.12}$, respectively. Thus, the effective signal-to-noise ratio increases with increasing $V_{s w}$. The observations suggest that the noise generation is associated with reconnection processes.
\end{abstract}

Key words. Magnetospheric physics (magnetopause, cusp, and boundary layers; MHD waves and instabilities; solar wind magnetosphere interactions)

\section{Introduction}

Daytime variations of the geomagnetic field in the Pc3-4 frequency range $(\sim 10-100 \mathrm{mHz})$ are observed at all magnetic latitudes from the polar caps to the equator, and have been studied extensively in space and on the ground. Although

Correspondence to: P. V. Ponomarenko

(phpp@alinga.newcastle.edu.au) different research groups use different selection criteria and analysis techniques, several common features have been established for these pulsations. For example, Pc3-4 occurrence and power maximizes near 10:30-11:00 MLT (Engebretson et al., 1986; Morris and Cole, 1987; Morrison, 1991; Takahashi and Anderson, 1992; Cao et al., 1994) and increases with decreasing interplanetary magnetic field (IMF) cone angle (Gul'elmi et al., 1973; Greenstadt et al., 1979b; Wolfe et al., 1980; Takahashi et al., 1981; Yumoto et al., 1984). The early observation that Pc3-4 pulsation frequency increases with growing IMF magnitude, $\left|B_{\mathrm{IMF}}\right|$ (for the appropriate reference list, see detailed review of, Odera, 1986), suggests that at least some component of the pulsation spectrum is produced by an ion-cyclotron instability in reflected proton beams upstream of the bow shock (Fairfield, 1969; Paschmann et al., 1979; Hoppe and Russell, 1983; Yumoto, 1985).

On the ground, Pc3-4 power maximizes somewhere between 60-80 geomagnetic latitude (MLAT) (Fraser-Smith, 1982; Bol'shakova and Troitskaya, 1984), the exact latitude of peak power moving equatorward with increasing $K_{p}$ (Bol'shakova and Troitskaya, 1984). This has prompted speculation about the cusp and/or boundary layer as an effective channel for Pc3-4 energy transport from the magnetopause to the high-latitude ionosphere (Bol'shakova and Troitskaya, 1984; Engebretson et al., 1986). Engebretson et al. (1991) proposed an "ionospheric transistor" model, in which the magnetospheric-ionospheric current system is modulated either directly by incoming compressional Pc34 waves at the magnetopause, or indirectly via streams of precipitating particles modulated by ULF waves at the magnetopause. Other possible Pc3-4 generation/transmission mechanisms include (1) propagation of fast magnetosonic waves direct into the magnetosphere, (2) surface compressional waves at the magnetopause, and (3) harmonics of field line resonances (Yumoto et al., 1984). Previously published experimental results do not allow us to distinguish a dominant propagation mechanism to high latitudes, and more work has to be done in this direction. 
Besides upstream wave sources, some studies have also emphasized the contribution to the cusp-latitude ULF spectrum from broad-band noise (Engebretson et al., 1986, 1995; McHarg et al., 1995; Posch et al., 1999). It was established that the noise power increases with increasing solar wind velocity, but does not show any clear dependence on IMF parameters (Engebretson et al., 1995; Posch et al., 1999). However, to our knowledge, there has been no quantitative study on the relative contributions from upstream waves and broad band noise to total power in the high-latitude Pc3-4 band.

In this paper, we try to establish statistically significant characteristics of cusp-latitude Pc3-4 waves and their relationship to upstream parameters. This is achieved by analyzing three years of magnetometer records from a cusp-latitude ground station in Antarctica, providing improved confidence in the conclusions than would be the situation in a limited case study approach. The study comprised three phases. First, we analyzed instantaneous and average ULF spectra and identified the signatures of upstream waves and the underlying background spectrum. Second, we used this information to develop an analytical procedure for separating both of these components. Third, we then used this procedure to undertake separate studies of the seasonal and diurnal variations and dependence on the IMF and solar wind for these two components. These results provide new insights into the source and generation of Pc3-4 energy in the high latitude magnetosphere.

\section{Experimental data and preliminary processing}

In this study, we used induction magnetometer data from the Australian Antarctic station Davis (geographic $68.6^{\circ} \mathrm{S}$, $78.0^{\circ} \mathrm{E}$; geomagnetic $74.6^{\circ} \mathrm{S}, 102.3^{\circ} \mathrm{E}$ ). We chose this station because of its proximity to the daytime cusp/cleft regions considered by Engebretson et al. (1991) as the main Pc3-4 energy channel to the high-latitude magnetosphere.

The magnetometer database covered the three consecutive years, 1995-1997. The data were obtained from digital records of the two horizontal geomagnetic field components along and perpendicular to the direction to the dip pole. The sampling rate changed over the data set, but the lowest rate was $0.5 \mathrm{~Hz}$ (Nyquist frequency $0.25 \mathrm{~Hz}$ ). Absolute and relative calibration of the magnetometer was performed annually and daily, respectively, using internal and external calibration coils, as well as a rotating magnet. In addition, the low-frequency portion of the induction magnetometer output was compared with records from a co-located fluxgate magnetometer of known calibration.

Prior to processing, days with unreliable data were extracted from the database. These included days with large data gaps, artificial or instrumental interference, and analogto-digital conversion limiting. The total amount of data removed did not exceed 24 days/year and 7 consecutive days.

The induction coil sensors produce a frequency-dependent response, $(d B / d t) \propto f$, and to compensate for this over the Pc3-5 range, we integrated the data records in the time domain on a point-by-point basis. Validity of this procedure was confirmed by comparison of power spectra from "raw" and integrated time series. As expected, the ratio of these spectra was proportional to $f^{-2}$ within Pc3-5 frequency band.

Further data processing consisted of the calculation of power spectra using a conventional Fourier transform. For this purpose we used 30-min time intervals, weighted by a Hanning window with 50\% overlap. Only trace power density, which represents the sum of power spectra from both horizontal components, was analyzed. This parameter was used because it is invariant with respect to the coordinate system in the horizontal plane.

Interplanetary plasma parameters $\left(B_{\mathrm{IMF}}\right.$ and $\left.V_{S W}\right)$ were obtained from WIND satellite data, accessible via the Internet, courtesy of NASA/Goddard SFC. These data, sampled at $\sim$ a 90-s rate, were shifted in time to compensate for the propagation delay from the satellite position to the Earth's bow shock. They were then averaged over the same 30min intervals used for the magnetic data analysis. Additional analysis of the WIND data showed that the autocorrelation time for all IMF and SW parameters usually exceeds $30 \mathrm{~min}$, which supports our choice of the analyzed interval length.

\section{Components of the Pc3-4 spectrum}

In this section, we look for a simple and comprehensive signature of upstream-generated Pc3-4 wave power at high latitudes, based on their statistical characteristics.

\subsection{Band-limited Pc3-4 waves and their source.}

First, we looked through many instantaneous daytime spectra and found, quite subjectively, that narrow-band waves with $\Delta f / f_{\max } \ll 1$ are rather rare at high latitudes, where $f_{\max }$ is the frequency of the spectral maximum and $\Delta f$ the effective spectral width. However, almost every day bandlimited Pc3-4 activity can be observed. We illustrate this by considering spectra for a typical, apparently featureless day, 01 March 1996, presented in Fig. 1. The top panel shows a whole-day spectrogram over the $0-100 \mathrm{mHz}$ frequency range. This format is widely used (e.g. Engebretson et al., 1995), except that we have compensated for the $(d B / d t)$ response of the induction sensors, as explained above. In Fig. 1, the spectrogram indeed looks rather featureless and noisy, and this fact has been used in Engebretson et al. (1995) as a major argument for the domination of broad band noise over band-limited signals. However, a closer examination of selected power spectra plotted on log-log scales (the nine bottom panels; time shown corresponds to the center of the analyzed 0.5 -h interval) reveals the existence of a band-limited "bump" or enhancement somewhere between 10 and $100 \mathrm{mHz}$. This is indicated by the arrow in Fig. 1. The frequency and magnitude of this "bump" vary with time: sometimes it is not apparent as a spectral enhancement, but just appears as a flattening on a very steep power-law-like 

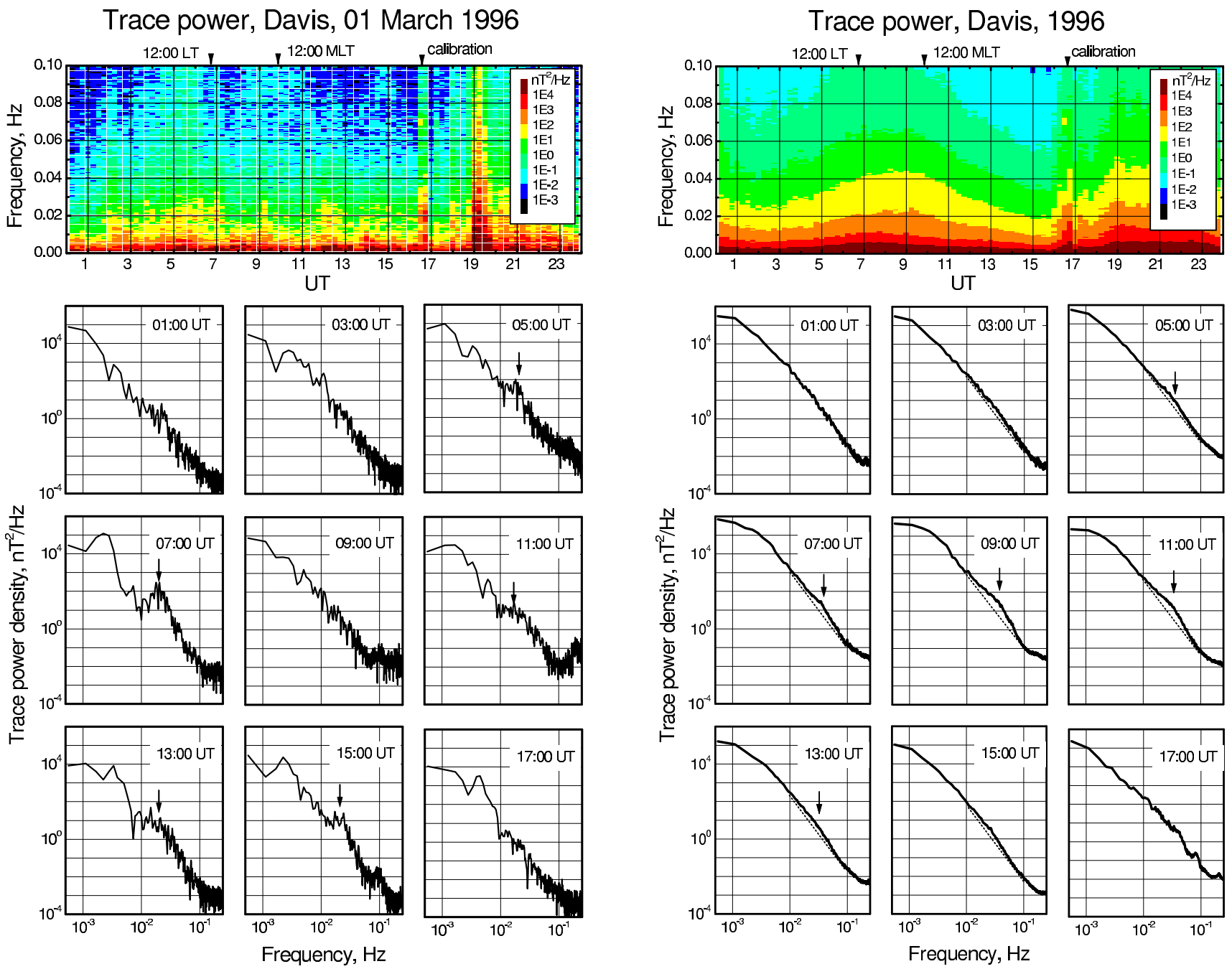

Fig. 1. Example of cusp latitude diurnal spectrogram (top panel) and selected power spectra (nine bottom panels) for 01 March 1996. Arrows show approximate positions of band-limited spectral enhancements ("bumps") in Pc3-4 range.

background (e.g. 11:00 and 13:00 UT spectra in Fig. 1); but usually it comprises several sub-maxima superimposed on the background spectral slope (05:00, 07:00, 15:00 UT).

The other noticeable features of the spectrogram in the top panel in Fig. 1 are a maximum in broad band nighttime power connected with substorm activity from 18:00-20:00 UT, and a field line resonance (FLR) maximum around $2 \mathrm{mHz}$ that is better seen in the power spectra.

To continue with our intention to analyze statistical data rather than individual events, Fig. 2 presents the whole-day spectrogram and selected power spectra averaged over the entire year of 1996, in the same format as Fig. 1. The diurnal spectrogram in the top panel exhibits a pre-noon ( 11:00 MLT) maximum in activity, spanning the entire frequency range between 0 and $100 \mathrm{mHz}$. However, inspection of the power spectra clearly shows that the band-limited "bump" is still present. In each panel, a dotted line repre-

Fig. 2. Average diurnal spectrogram and power spectra for all of 1996 in the same format as Fig. 1. Line thickness of the power spectra correspond to the $95 \%$-confidence limits. Dotted lines represent $S \propto f^{-4}$.

sents the power-law background spectrum (discussed later), and the band-limited flattening of the spectral slope is labelled by an arrow. Smoothed and broadened due to averaging, the "bump", nevertheless, remains noticeable above the background between 05:00-13:00 UT, with maximum power density around 09:00 UT, i.e. 11:00 MLT. Line thickness in the power spectra in Fig. 2 represents the 95\%-confidence interval for the given statistics (332 averaged instantaneous spectra) $0.86 S(f)<S(f)<1.17 S(f)$, where $S(f)$ is a power spectral density estimate.

To clarify the origin of this band-limited enhancement, we examined the dependence of ULF spectra on $\left|B_{\mathrm{IMF}}\right|$ during local daytime (03:00-13:00 UT) over all of 1996. In doing this, we sorted the ground magnetometer data into $1 \mathrm{nT}$ bins with respect to IMF magnitude. Four of the resultant spectra are presented in Fig. 3. To facilitate comparison the spectra are shifted from each other by an order of magnitude. To 




Fig. 3. Dependence of spectral shape on $\left|B_{\mathrm{IMF}}\right|$ at Davis over 1996. Error bars under each curve denote respective $95 \%$-confidence intervals. Dotted lines represent $S \propto f^{-4}$.

illustrate the significance of the spectral "bump", we indicate the corresponding 95\%-confidence intervals below each spectral curve. The relative sizes of these error bars change from curve to curve, due to the different numbers of averaged instantaneous spectra. The spectral "bump" clearly moves toward higher frequencies with increasing $\left|B_{\mathrm{IMF}}\right|$, and, therefore, we conclude that this band-limited enhancement represents a signature of waves generated in the upstream IMF. Henceforth, we refer to this band-limited "bump" or enhancement as a signal. The signal is hardly noticeable in the bottom curve ( $1-2 \mathrm{nT})$, where it seems to be obscured by relatively large statistical variations in $S(f)$, resulting from fewer averaged spectra for these values of $\left|B_{\mathrm{IMF}}\right|$.

The bottom curve in Fig. 3 also allows us to estimate the spectral shape of the background noise component. In this spectrum, the signal ("bump") is moved to the lower Pc4 range, and the remaining spectral density for the background noise may be represented quite accurately by a power law of the form $S_{n}(f) \propto f^{p}$, where $p \simeq-4$. Due to its broadband nature, henceforth, we refer to this component as noise. The existence and importance of such a power-law component at cusp latitudes has also been emphasized by others (e.g. Olson, 1986; Engebretson et al., 2000).

Regarding power spectra from Figs. 1, 2, and 3, it is neces- sary to point out an important property of logarithmic scale - its relative nature. For example, in the power spectrum for 11:00 UT in Fig. 2, the apparent maximum difference between the noise (dashed line) and total spectral density (solid line) is denoted by an arrow at around $30 \mathrm{mHz}$. This occurs at frequency $f_{0}$. In contrast to a linear scale, this does not necessarily mean that the signal has its maximum there too. In fact, this apparent difference depends on a ratio between total spectral power $S(f)$ and noise $S_{n}(f)$ :

$$
\begin{gathered}
\ln S(f)-\ln S_{n}(f)=\ln \left(S(f) / S_{n}(f)\right)= \\
\ln \left(S_{S}(f) / S_{n}(f)+1\right)=\ln (R(f)+1),
\end{gathered}
$$

where $S_{s}(f)$ and $R(f)$ are the signal spectrum (bump) and signal-to-noise ratio spectrum, respectively. As a result, the logarithmic scale effectively emphasizes the signal-to-noise ratio rather than the signal level itself, and requires cautious interpretation.

\subsection{Separation algorithm}

We now use information obtained in the previous section to deconvolve the signal and noise components of the cusplatitude Pc3-4 spectrum. The various steps in this procedure are illustrated in Fig. 4, which represents a part of the average spectrum for 09:00 UT (Fig. 2) between 10 and $100 \mathrm{mHz}$.

First of all, it is necessary to estimate power-law noise parameters. Engebretson et al. (2000) used for this purpose a noise slope and magnitude measured from spectra averaged over the whole daytime. Obviously, if these parameters vary with time, this may lead to under or overestimates of the noise contribution to each particular spectrum, especially when $R \simeq 1$. In our work, we estimated the noise parameters for every power spectrum in the following way. Looking at the average spectra in Fig. 2, the noise usually dominates the signal at the extremes of the $10-100 \mathrm{mHz}$ frequency range. We used this fact for estimating the slope $p$ and magnitude $S_{n 0}^{\text {mod }}$ for the model powerlaw noise spectrum $S_{n}^{\text {mod }}(f)=S_{n 0}^{\text {mod }} \cdot f^{p}$ (henceforth, the superscript ${ }^{\text {mod }}$ will depict model spectral characteristics to distinguish them from the experimentally measured ones). Technically, the procedure consisted of fitting a linear function to the $\log \{S[\log (f)]\}$ dependence between $10-15$ and $80-100 \mathrm{mHz}$. Figure 4a shows the input "raw" experimental spectrum $S(f)$ (circles) and a fitted noise model (dashes).

There have been several previous attempts to reduce the effect of power-law noise on magnetometer data (e.g. Takahashi et al., 1984; Engebretson et al., 2000). The most frequently used procedure is based on the so-called "prewhitening", which involves normalizing the spectral density by a model noise slope $f^{p}$ and converting the coloured noise into white. Similar to the logarithmic scale discussed in the previous section, this algorithm is quite effective in emphasizing spectral components with high signal-to-noise ratios. Actually, induction magnetometers with their amplitude response $(d B / d t) \propto f\left(\propto f^{2}\right.$ for power) compensate, at least partially, for the power-law background of the geomagnetic 


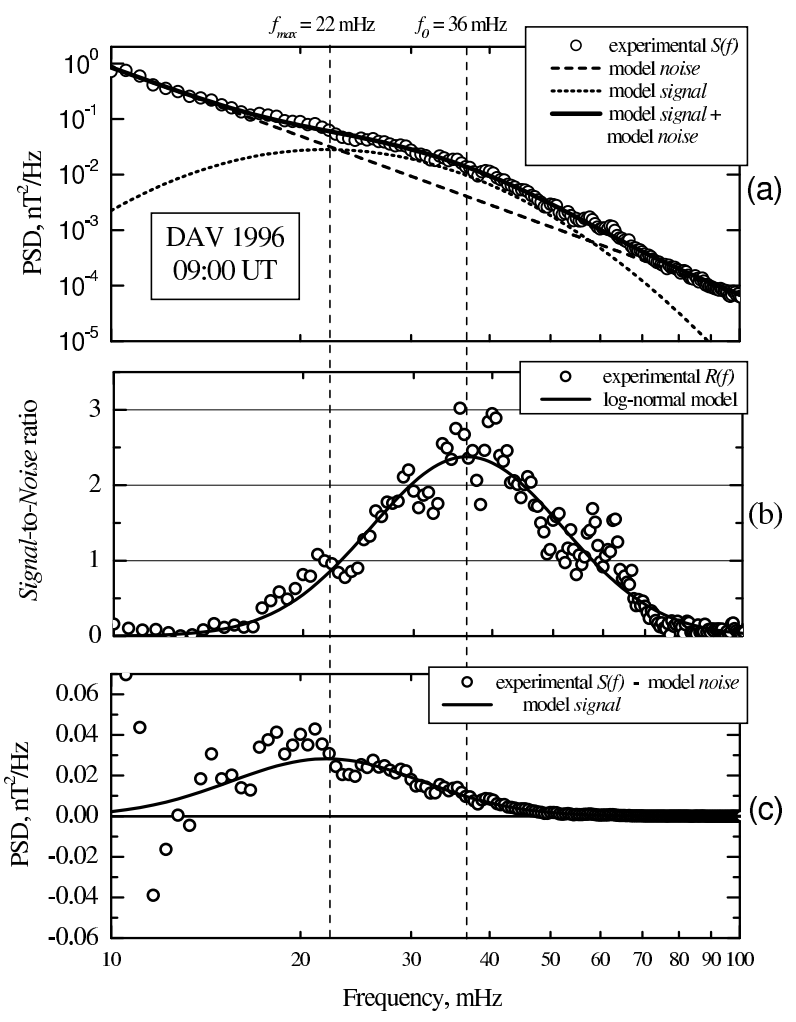

Fig. 4. Experimental (circles) and fitted model (solid line) spectra: (a) total power signal + noise, (b) signal-to-noise ratio, and (c) signal power obtained for the power spectrum for 09:00 UT in Fig. 2. Dashed and dotted lines in (a) show model noise and signal power, respectively. $f_{0}$ indicates the frequency where the observed signal-to-noise ratio reaches a spectral maximum; $f_{\max }$ is the frequency where the signal has maximum PSD.

field. However, "pre-whitening" also distorts the true spectral density distribution in the frequency domain and must, therefore, be treated with caution. To illustrate this, let us represent a measured ULF spectrum in the Pc3-4 band as the sum of band-limited signal, $S_{s}$, and power-law noise, $S_{n}$ :

$S(f)=S_{S}(f)+S_{n}(f)=S_{s}(f)+S_{n 0} f^{p}$,

where $S_{n 0}=$ const and $p<0$. After pre-whitening, the noise level will be uniform and equal to $S_{n 0}$, but the signal spectrum will be strongly distorted $\propto f^{-p}$ :

$S(f) f^{-p}=S_{s}(f) f^{-p}+S_{n 0}$.

This shifts the resulting spectral maximum toward higher frequencies and results in an underestimate of the lower frequency contribution to the integral power. If $S_{n 0}$ is known, it becomes possible to restore a signal-to-noise ratio from the following equation:

$S(f) / S_{n}(f)=S_{s}(f) / S_{n}(f)+1=R(f)+1$

(compare with Eq. 1). Open circles in Fig. 4b represent an experimental estimate of $R(f)$ based on the substitution of

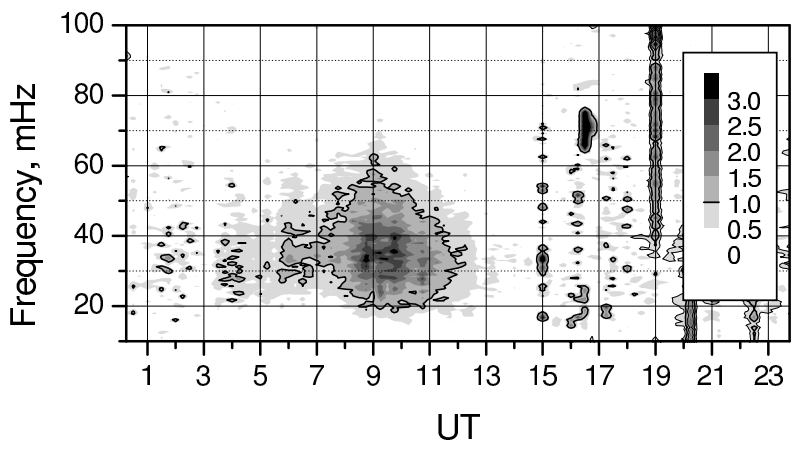

Fig. 5. Diurnal spectrogram of the signal-to-noise power ratio $R$ at Davis over 1996, calculated for the spectrogram in the top panel of Fig. 2. A solid line contour corresponds to $R=1$.

the fitted noise model into Eq. (3). Also, this equation allowed us to construct an average diurnal spectrum of the signal-to-noise ratio based on the spectrogram from Fig. 2 . The result is shown in Fig. 5, which shows that, on average, the signal at Davis prevails over the noise between 07:00 and 12:00 UT and in the frequency range $\sim 20-60 \mathrm{mHz}$.

Another more correct and comprehensive approach may be based on extracting $S_{n}^{\text {mod }}(f)$ from the experimental spectrum $S(f)$ (Engebretson et al., 2000). The signal spectrum, estimated in this way, $S_{s}(f)=S(f)-S_{n}^{\text {mod }}(f)$, is shown by open circles in Fig. 4c. However, in this situation, another difficulty arises from the coloured nature of the noise. Averaged spectra always contain statistical variations of spectral density, whose magnitude $\delta S(f)$ decreases with the number of averagings but never reaches zero. If we just extract a power-law slope from $S(f)$, these variations will still remain in the reconstructed signal spectrum and will prevail in regions with $R \ll 1$. In our case, $\delta S(f)$ increases with $f \rightarrow 0$ (Fig. 4c), which one would expect, taking into account that the noise power and, consequently, its variance is proportional to $f^{-4}$. This component may be easily confused with the signal proper and causes serious problems in measuring the signal spectrum shape, especially the position of the peak, $f_{\text {max }}$. Also, direct extraction of $S_{n}^{\text {mod }}(f)$ from the experimental spectra may result in physically meaningless negative values of spectral density for $R(f) \ll 1$. Both these effects are clearly seen in Fig. $4 \mathrm{c}$ for $f \leq 20 \mathrm{mHz}$.

To be able to obtain a smooth and meaningful estimate of the signal spectrum, we applied a model fit $R^{\bmod }(f)$ for the experimentally measured signal-to-noise ratio defined by Eq. (3), and then restored the model signal spectrum via a "de-whitening" procedure

$S_{s}^{\text {mod }}(f)=R^{\text {mod }}(f) \cdot S_{n}^{\text {mod }}(f)$.

The advantage of this procedure is that the major contribution to the fitted model is given by the spectral component with the highest $R(f)$, and contributions from statistical variations of the noise at both low- and high-frequency ends of the analyzed frequency range $10-100 \mathrm{mHz}$ are minimized and equalized by pre-whitening. As one can see from Fig. 4b, 
the measured $R(f)$ (circles) can be approximated quite well by a log-normal model (solid line)

$$
R_{n}^{\text {mod }}(f)=R_{0}^{\text {mod }} \exp \left(-\left(\ln \left(f / f_{0}\right)\right)^{2} / \sigma_{f}^{2}\right) .
$$

Here, $R_{0}^{\bmod }=$ const $>0$ is a maximum value of $R^{\bmod }(f)$ at frequency $f_{0}$, and $\sigma_{f}$ is a dimensionless parameter defining an effective width of the log-normal function. The effective width at $1 / e$ level is

$\Delta f=f_{0} \cdot \sinh \sigma_{f}$

The restored model signal spectrum (Eq. 4) is represented by the solid line in Fig. 4c. Comparison with Fig. 4b illustrates the above-mentioned important side effect of prewhitening: a shift of the spectral maximum toward higher frequencies observed in $R(f)$ with respect to $S_{S}(f)$. This shift may be considerable; in our case, for $R_{0} \simeq 2.5$, it is more than $10 \mathrm{mHz}$ - from $f_{\text {max }}=22 \mathrm{mHz}$ to $f_{0}=36 \mathrm{mHz}$. In general, this may cause a large bias in estimating $f_{\text {max }}$ from all kinds of pre-whitened data, including induction magnetometer records not compensated for $(d B / d t)$.

It is easy to show that multiplication of the log-normal function by a power-law one (Eq. 4) results in another lognormal function with the same $\sigma_{f}$, but with frequency maximum at

$f_{\max }=f_{0} \cdot \exp \left(p \sigma_{f} / 2\right)$.

Due to the negative value of $p \simeq-4$, this leads to $f_{\max }<f_{0}$, i.e. to smaller values of the spectral maximum frequency for the signal, in comparison with that for $R(f)$. Note that both "whitening" the noise $(p \rightarrow 0)$ and narrowing the signal spectral width $\left(\sigma_{f} \rightarrow 0\right)$ cause $f_{\max } \rightarrow f_{0}$.

However, the described signal and noise separation procedure may only be used to analyze spectra in which $f_{0}$ is not close to 10 or $100 \mathrm{mHz}$, and where the condition $R \ll 1$ is satisfied within the $10-15$ and $80-100 \mathrm{mHz}$ frequency ranges. Otherwise, the noise and, consequently, the signal parameters would be calculated incorrectly. This is the case when the IMF magnitude is particularly low or high (top and bottom curves in Fig. 3). In such situations, the slope was simply fixed at $p=-4$, and instead of using a log-normal model for $R(f)$, we used the same model but with a constant offset $S_{n 0}$ for a pre-whitened spectrum (Eq. 2). The same also applies to the IMF $B_{z}$-dependence discussed later, and the calculation of the power law parameter $p$ discussed in the next section.

Of course, the above algorithm is not perfect for estimating $f_{\text {max }}$, taking into account that the signal spectral maximum lies in a frequency range with $R \simeq 1$ (Figs. $4 \mathrm{~b}$ and c). However, it is the best available in this situation.

Finally, to demonstrate the reliability of the developed separation algorithm, we reconstructed a total power spectrum from the signal and noise models

$$
S^{\text {mod }}(f)=S_{s}^{\text {mod }}(f)+S_{n}^{\text {mod }}(f)
$$

and overlayed it onto the "raw" experimental spectrum $S(f)$ in Fig. 4a (solid line and circles, respectively). The dotted line in this figure corresponds to $S_{s}^{\text {mod }}(f)$.

In summary, our data processing algorithm presents a modified combination of previously developed prewhitening and noise extraction techniques (Takahashi et al., 1984; Engebretson et al., 2000). It consists of the following stages:

1. Calculating the power spectrum using the Fourier transform;

2. Determining the noise slope $p$ by fitting a linear function to the $\log \{S[\log (f)]\}$ dependence at the edges of the $10-100 \mathrm{mHz}$ frequency range, where $R \ll 1$ (excluding $\left|B_{\mathrm{IMF}}\right|$ and $B_{z}$ dependence when $p$ was assumed equal to -4$)$;

3. Establishing a signal-to-noise spectrum $R(f)$ by normalizing the experimental spectrum with the model noise determined in stage 2 ;

4. Fitting a log-normal model $R^{\bmod }(f)$ to this spectrum;

5. Reconstructing (de-whitening) the signal spectrum via multiplying $R^{\text {mod }}(f)$ by a model representing the noise;

6. Estimating the noise and signal parameters from the model functions separately.

The new elements we have introduced are the following:

- estimating the noise parameters for each experimental power spectrum $S(f)$ by fitting a power-law model to spectral regions with low $R(f)$;

- using a log-normal model for the signal-to-noise ratio to compensate for the "coloured" nature of statistical fluctuations in $S(f)$;

- obtaining a signal spectrum by de-whitening the fitted model for $R(f)$ (Eq. 4).

\section{Experimental results}

Here, we show results obtained for the band-limited signal and power-law noise at Davis over 1995-1997. We also examined the power of Pc5 ULF waves at Davis, which are believed to be driven by a Kelvin-Helmholtz instability at the magnetopause (see, e.g. Samson, 1972), as a reference for establishing the source of the Pc3 noise. When calculating integrated trace power, we used the frequency bands of 22.2$100 \mathrm{mHz}$ for both Pc3 signal and noise, and $1.67-6.67 \mathrm{mHz}$ for Pc5 waves, in order to be compatible with previous studies (e.g. Olson, 1986; Morris and Cole, 1987). Analysis of the signal curve in Fig. 4c shows that up to one third of the total signal power may lie in the Pc4 range below $22 \mathrm{mHz}$. However, to maintain the signal-to-noise ratio above 1 , we 



Fig. 6. Diurnal variation in integrated ULF trace power at Davis over 1995-1997, plotted against magnetic local time for (a) Pc3 signal, (b) Pc3 noise, (c) and Pc5 components; and (d) noise slope.

decided to use this frequency as a lower integration limit, because around it $R$ exceeds unity (Figs. $4 \mathrm{~b}$ and 5). Choice of the high-frequency boundary ( $100 \mathrm{mHz}$ in our case) is not so important due to the much lower spectral power level. Also, we compared the integrated Pc3 signal power obtained from the model (Eq. 4) and from the raw spectrum $S_{s}(f)$ (see, for example, solid line and circles in Fig. 4c) and found a negligible difference. The major reason we used this model was to be able to estimate $f_{\max }$.

The diurnal variation in power for different components of the ULF spectra are presented in Fig. 6. We are interested only in daytime activity and do not consider substorm associated pulsations before 03:00 and after 17:00 MLT. Fig. 6a shows signal power integrated over $22-100 \mathrm{mHz}$ as a function of magnetic local time. This component exhibits a clear maximum $1-2 \mathrm{~h}$ before local magnetic noon, as also seen for 1996 in Fig. 2. In contrast, the noise (Fig. 6b) reaches a maximum earlier in the morning sector. The total Pc5 power (Fig. 6c) shows a very similar trend to that for Pc3 noise. The variation of the noise slope is shown in Fig. 6d. This is almost constant across local day time with $p \simeq-4$.
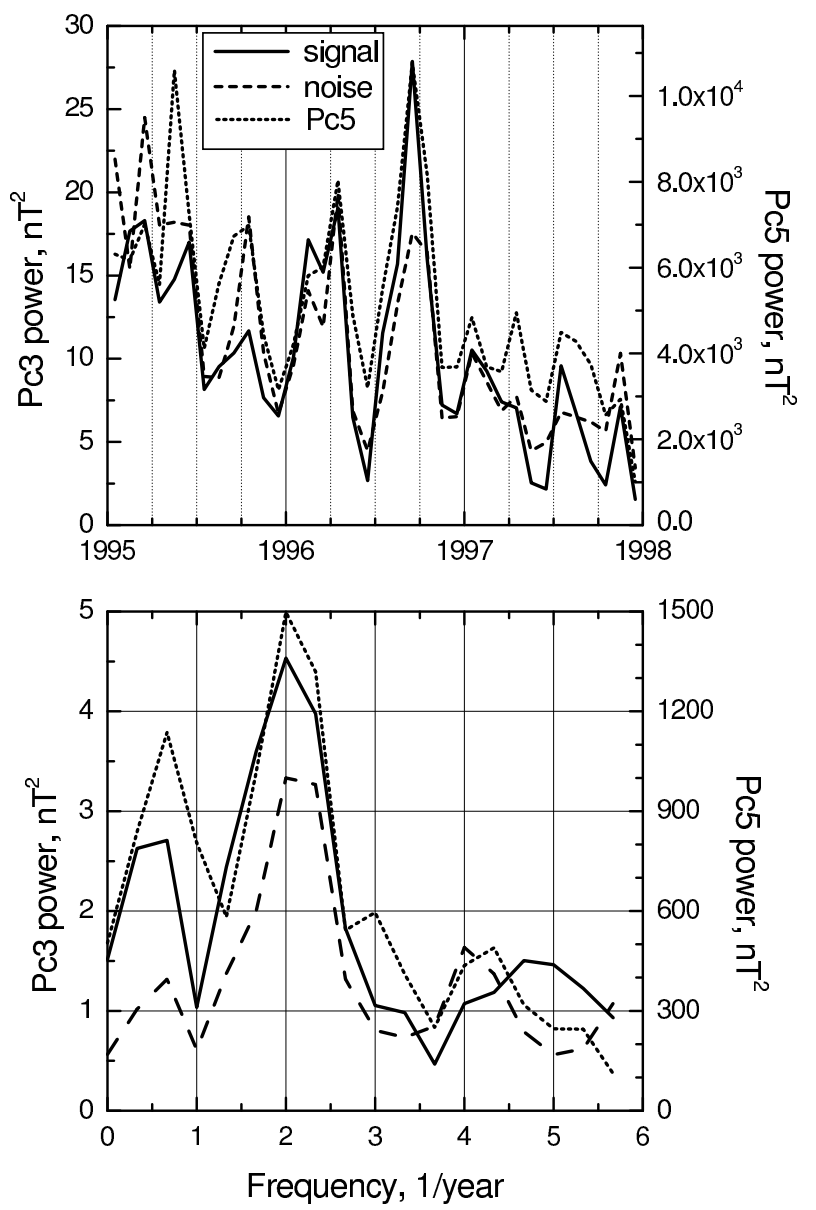

Fig. 7. Annual variations in integrated trace power at Davis over 1995-1997 (top) and corresponding frequency spectra (bottom).

Fig. 7 shows the annual variation in trace power for Pc3 signal and noise and Pc5 pulsations integrated over 03:0013:00 UT ( 05:00-15:00 MLT). The time series (top panel) exhibit a semiannual variation in all components, with maxima around the equinoxes and minima in the winter and summer months, especially for 1996 . This pattern is not so clear for 1995 and 1997, but the corresponding Fourier spectra (bottom panel) confirm the existence of the semiannual periodicity by showing pronounced coincident maxima at $f=2 \mathrm{yr}^{-1}$.

As mentioned in Sect. 3.2, the use of a signal interpolation model allows us to estimate the frequency of the spectral maximum $f_{\max }$. Open circles and triangles in Fig. 8 show the experimental dependence of $f_{\max }$ on $\left|B_{\mathrm{IMF}}\right|$ over 19951997. A linear fit to the average (solid line) gives $f_{\max }[\mathrm{mHz}]$ $=(-0.50 \pm 1.16)[\mathrm{mHz}]+(4.41 \pm 0.25)\left|B_{\mathrm{IMF}}\right|[\mathrm{nT}]$. For comparison, the dashed line in Fig. 8 represents the same dependence for the signal-to-noise ratio maximum frequency $f_{0}$ with a linear fit $f_{0}[\mathrm{mHz}]=(9.99 \pm 1.06)[\mathrm{mHz}]+(4.54 \pm$ $0.22)\left|B_{\mathrm{IMF}}\right|[\mathrm{nT}]$. Despite having virtually the same slope as for $f_{\max }$, this dependence exhibits a statistically significant bias $\simeq 10 \mathrm{mHz}$ toward higher frequencies, due to the prewhitening described in a previous section. 


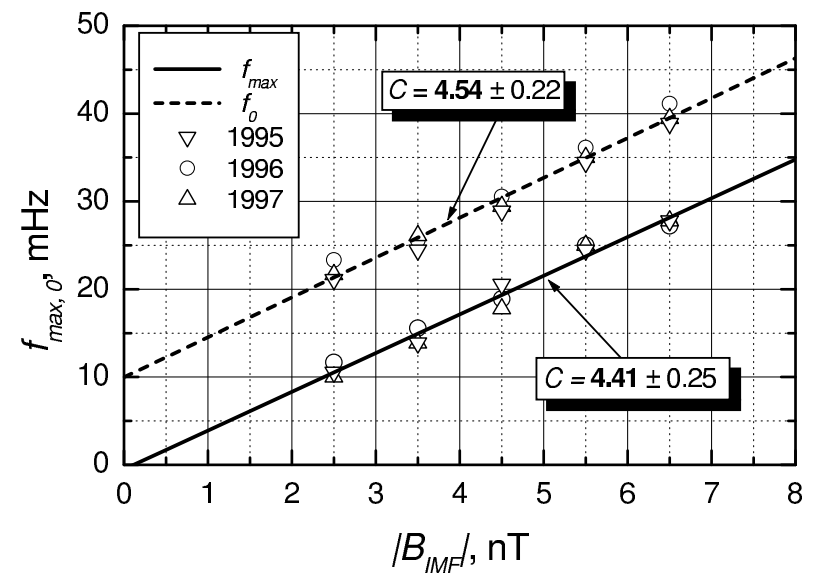

Fig. 8. Experimental dependence of spectral maximum frequency (circles and triangles) on IMF magnitude at Davis over 1995-1997 and corresponding linear fit lines. The solid line represents an estimate for the band-limited signal $\left(f_{\max }\right)$, and the dashed line corresponds to that for the signal-to-noise ratio $\left(f_{0}\right)$.

To examine the dependence of the signal and noise parameters on solar wind cone angle, we used $\theta_{x B}=$ $\cos ^{-1}\left(B_{x} /\left|B_{\mathrm{IMF}}\right|\right)$, where $B_{x}$ is the $B_{\mathrm{IMF}}$ component along a Sun-Earth line, which defines $\theta_{x B}$ between 0 and $180^{\circ}$. The signal (Fig. 9a) shows maximum power at $\theta_{x B} \simeq 20^{\circ}$ and $160^{\circ}$ and a deep minimum around $90^{\circ}$. In contrast, the noise power (Fig. 9b) is more uniformly distributed with $\theta_{x B}$, exhibiting only a slight decrease around $0^{\circ}$ and $180^{\circ}$. Pc5 power (Fig. 9c) demonstrates two symmetric maxima around $30-40^{\circ}$ and $140-150^{\circ}$ for 1995 and 1996, and an almost uniform dependence for 1997. The noise slope (Fig. 9d) exhibits a pronounced decrease from $p \simeq-4$ for $\theta_{x B} \simeq 30^{\circ}$ and $140^{\circ}$ to $p \simeq-4.5$ for $\theta_{x B} \rightarrow 0$ or $180^{\circ}$.

We also studied the dependence of pulsation trace power on the north-south component of the IMF $B_{z}$ (GSM). The results are presented Fig. 10 in the same format as in Fig. 9. The signal (Fig. 10a) has maximum power around $B_{z}=0$ and decreases with decreasing $\left|B_{z}\right|$. It is easy to explain this by taking into account the cone angle dependence from Fig. 9a, since with increasing $\left|B_{z}\right|$ the cone angle approaches $90^{\circ}$, where there is a deep minimum in the band-limited power.

The noise component (Fig. 10b) is assumed to have a constant slope with $p=-4$, and exhibits a nearly uniform distribution for $B_{z}>0$ but increases when $B_{z}<0$. This suggests that at least part of the noise energy may depend in some way on reconnection processes at the magnetopause. The Pc5 component in Fig. 10c is invariant to the polarity of $B_{z}$ and appears rather uniform.

The dependence of ULF power on solar wind velocity for all values of $B_{z}$ is shown in Fig. 11a, together with the corresponding power-law fits for $\beta$, where Power $\propto V_{s w}^{\beta}$. The band-limited signal exhibits the steepest slope with $\beta=5.71 \pm 0.26$, followed by the noise $(\beta=4.12 \pm 0.22)$, then the $\operatorname{Pc} 5$ ( $\beta=3.44 \pm 0.19)$ components. Figure $11 \mathrm{~b}$
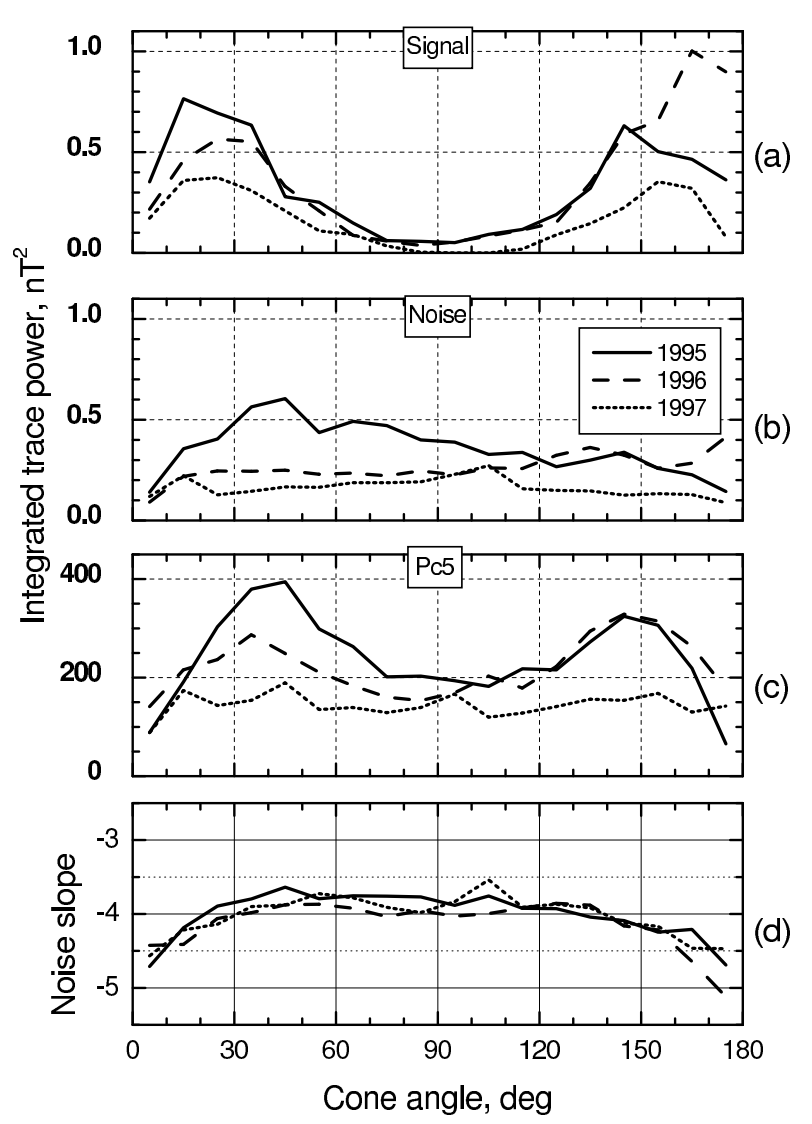

Fig. 9. Cone angle dependence at Davis over 1995-1997 for (a) Pc3 signal, (b) noise, (c) Pc5 trace power, and (d) noise slope.

presents the same dependence but only for $B_{z}>0$, i.e. the effects of reconnection are excluded. This leads to an increase in $\beta$ for the noise and $\operatorname{Pc} 5$ by $\sim 0.5$, while there is no significant change in the signal slope.

\section{Discussion}

The major goal of this work has been to quantitatively analyze the morphological structure of high-latitude Pc3-4 spectra and compare this with the better understood Pc5 spectra. This section compares our experimental results with previous studies and emphasizes the significance of the newly obtained information on Pc3 band-limited signal and powerlaw noise components.

\subsection{Separation of signal and noise}

Many previous authors have discussed the form of the ULF spectrum at Pc3-4 frequencies. It is generally accepted that at local daytime band-limited power enhancements in the Pc3-4 range are superimposed upon power-law background. Determination of the exact form of the spectral contributions has been complicated by the different analytical approaches used in previous experimental studies. Simple and compre- 


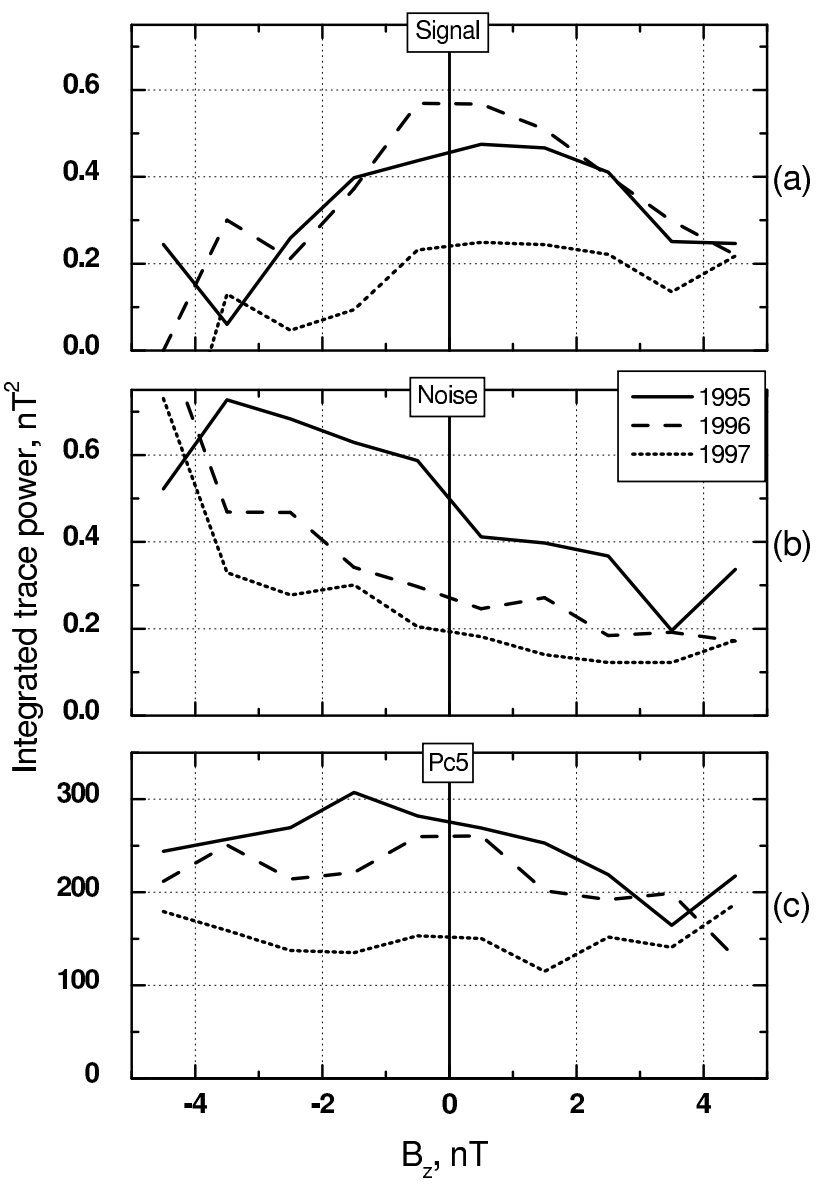

Fig. 10. Dependence of integrated trace power at Davis over 19951997 on $B_{z}$ for (a) signal, (b) noise, and (c) Pc5 component.

hensive selection and recognition criteria are required when comparing observations.

Our approach to this problem involves examining spectra averaged over a large database, from which we deconvolved a broad band background power-law component that we call noise, and a band-limited component that we call signal. These labels are purely descriptive and are not meant to specify generation mechanisms or wave modes. Our approach is justified by the observation that the signal and noise components exhibit different diurnal, solar wind and IMF dependences. The noise follows a power law of the form $S_{n}(f) \propto f^{-4}$, while the amplitude and center frequency of the signal peak depends on the orientation and magnitude of the IMF, respectively.

Our findings are supported by Wolfe et al. (1980); Bol'shakova and Troitskaya (1984); Engebretson et al. (1986); Olson (1986); Morris and Cole (1987); Wolfe et al. (1987); Yumoto et al. (1987); Slawinski et al. (1988); Engebretson et al. (1990); McHarg et al. (1995), and Engebretson et al. (2000), who discussed the appearance of an IMF-dependent enhancement in cusp latitude ground spectra. Also, Takahashi and Anderson (1992) presented averaged spectra from the AMPTE CCE spacecraft over $L=2.5-6.5$

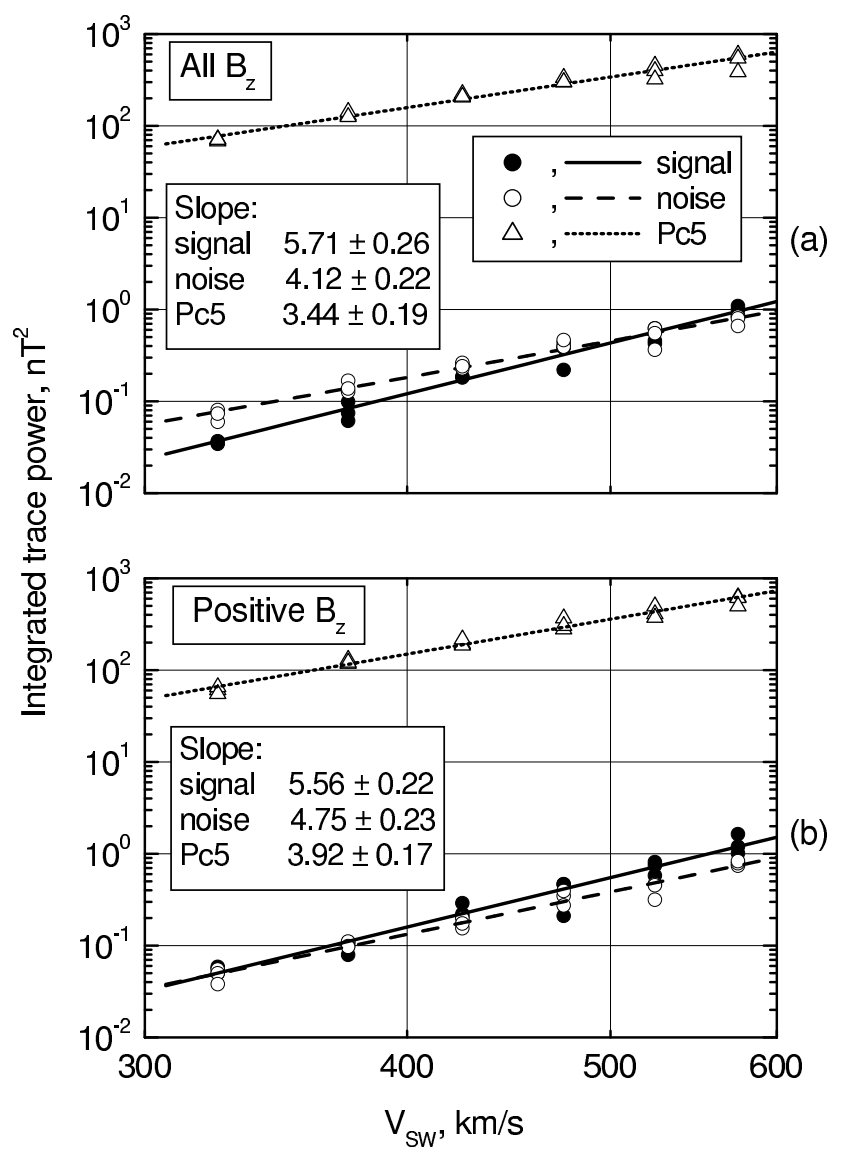

Fig. 11. Dependence of integrated trace power at Davis over 19951997 on solar wind velocity (a) for all $B_{z}$ and (b) for $B_{z}>0$.

within $\pm 30^{\circ}$ MLAT. Despite being acquired in space and far away from the cusp regions, their results show the characteristic band-limited "bump" between 10 and $100 \mathrm{mHz}$ very similar to what we have found in our work.

With respect to the broad band noise, Olson (1986) obtained a power law index of $p \simeq-2.6$ for average ground magnetometer spectra from a cusp latitude station at Cape Perry, Canada. Later, however, Posch et al. (1999) showed (their Fig. 6) a few examples of instantaneous noise spectra from the same station with $p \sim-(3.5-4)$, which is consistent with our results. Wolfe et al. (1994) examined hourly magnetometer spectra from two cusp stations for the 8 quietest days in 1989 , and found that over the $10-50 \mathrm{mHz}$ range the spectra could be described by a power law typically in the range of -2 to -5 , with spectral slopes less than -3 being common. Our results are also supported by a statistical study of DeLauretis et al. (1991), who found that the average daytime background spectra at cusp latitudes (Iqualuit, Canada) exhibit $p=-(3.47-3.80)$ over the Pc3 frequency range. As has been emphasized by Menk et al. (1993), these values cannot be explained solely by either the KHI, which predicts $p=-5 / 3$ (Kolmogoroff's hydrodynamic turbulence) or $p=-3$ (ion-acoustic turbulence), or the drift wave insta- 
bility giving $p=-5$ (D'Angelo, 1973).

As noted in Sect. 1, Engebretson et al. (1995) found a dominance of broad band noise at cusp latitudes. Their conclusion was based on visual inspection of different types of diurnal ULF spectra, and no quantitative study was attempted. In contrast, the current study directly measured $R$. The results obtained show that the Pc3 signal prevails over noise throughout the $\pm 2.5-3 \mathrm{~h}$ interval around local magnetic noon (Fig. 5).

\subsection{Diurnal and seasonal variations}

Diurnal variations of the band-limited Pc3 signal power shown in Fig. 6 are in agreement with previous results for cusp latitudes (Engebretson et al., 1986; Morris and Cole, 1987; Wolfe et al., 1987). A new finding is the similarity between the diurnal distributions of broad band noise and Pc5 power.

Equinoctial maxima in overall magnetic activity were first reported in the 19th century; however, their origin is still unclear (for the latest developments and appropriate references see, e.g. Cliver et al., 2000). We have observed a similar periodicity in the band-limited Pc3 signal component, which, to our knowledge, is a new result. It is intriguing that, despite their very different sources and diurnal properties, both Pc5 and Pc3 waves exhibit almost identical seasonal variations.

Another pronounced feature shown in Fig. 7 is the relatively low overall level of ULF activity during 1997. This fact is also reflected in Figs. 6, 9, and 10. Taking into account the strong dependence of all of the ULF spectral components on $V_{s w}$, it might be due to a lower average value of $V_{s w}$, which, according to our calculations, is $\left\langle V_{s w}\right\rangle \simeq 380 \mathrm{~km} / \mathrm{s}$ for 1997, in comparison with $420-430 \mathrm{~km} / \mathrm{s}$ for $1995-1996$. Estimates based on slopes from Fig.11 give us a corresponding decrease of $\simeq 0.55,0.65$, and 0.70 for signal, noise, and Pc5 power during 1997, respectively, which is in reasonable agreement with our results.

\subsection{Cone angle}

The symmetric dependence of Pc3 signal power on cone angle (Fig. 10a) shows that there is no preference for inward or outward $\mathbf{B}_{\mathrm{IMF}}$ direction, allowing us to consider only the interval between 0 and $90^{\circ}$. In general, our results agree with previous studies that showed maximum Pc3-4 occurrence and power for low cone angles, and minimum power for $\theta_{x B} \rightarrow 90^{\circ}$ (Wolfe et al., 1980; Takahashi et al., 1981; Russell et al., 1985; Yumoto et al., 1987; Morrison, 1991). This agrees with the interpretation proposed by Russell et al. (1985), who considered the effect of magnetosheath convection on Pc3-4 propagation from the bow shock to the magnetopause. Their scenario assumed that the most favourable entry region for the upstream waves lies in the vicinity of the stagnation point (i.e. low values of $\theta_{x B}$ ).

However, our observation that signal power decreases rapidly for lowest $\theta_{x B} \rightarrow 0$, also reported by Takahashi et al. (1981) using ATS-6 satellite data, somewhat contradicts the
Russell et al. (1985) model. It is unclear whether this observed $20-30^{\circ}$ offset of the signal power maximum from the Sun-Earth direction results from propagation or generation properties of the Pc3-4 waves. Also, when comparing our results with those obtained by other authors, it is necessary to keep in mind the distinction between integrated ULF power (that we have used) and event occurrence. Occurrence depends not only on power, but also on the signal-to-noise ratio and event selection criteria.

In contrast, the noise power varies little with cone angle. This argues against the noise source being waves entering from the upstream solar wind or generated on the flanks of the magnetopause. The slight increase in the noise slope for $\theta_{x B} \rightarrow 0$ or $180^{\circ}$ (Fig. 9) presents another interesting observation. However, it does not affect our average estimate of $p \simeq-4$ used for estimating $f_{\max }$, due to the low occurrence probability of very low cone angles (discussed below).

\subsection{IMF magnitude}

In agreement with previous authors, we find that the bandlimited Pc3-4 signal shows a nearly linear increase in frequency with increasing IMF magnitude:

$f[\mathrm{mHz}]=C\left|B_{\mathrm{IMF}}\right|[\mathrm{nT}]$.

However, our averaged data show $C=(4.42 \pm 0.25)$, in contrast to the frequently cited empirical value $C \simeq 6$ (Yumoto et al., 1984; Yumoto, 1985; Engebretson et al., 1986; Morris and Cole, 1987; McHarg et al., 1995). To explain this difference, we refer to an analytic formula derived in Takahashi et al. (1984), which also accounts for the cone angle dependence:

$f[\mathrm{mHz}]=7.6\left|B_{\mathrm{IMF}}\right|[\mathrm{nT}] \cos ^{2}\left(\theta_{x B}\right)$.

Our statistical approach is different from that used in most previous studies, where authors measured frequencies for selected events but not for average spectra over a long data set. Taking into account that upstream waves exhibit maximum amplitude for $\theta_{x B} \simeq 20-30^{\circ}$ (see Fig. 9a), most of the events examined in case studies would be drawn from this range of $\theta_{x B}$, which gives $C \simeq 5.7-6.7$, close to the empirical formula.

In contrast, our observations of the averaged spectral shape are also affected by the statistical distribution of the cone angle itself. This distribution is represented by the dark grey region in Fig. 12, and is a maximum at $\theta_{x B} \simeq 60^{\circ}$. Its convolution with the cone angle dependence for signal power (light grey) gives an effective average value $\left\langle\theta_{x B}\right\rangle \simeq 40^{\circ}$. This yields $C \simeq 4.5$, in reasonable agreement with our experimental data.

Another source of discrepancy may lie in using prewhitened data for estimating the position of the signal spectral maximum. As has been shown analytically in Eq. (5) and illustrated by real data in Figs. 4, 8, in this case the estimated frequency may have a considerable bias from $f_{\max }$ towards higher frequencies. The same applies to spectra presented using a logarithmic scale (see Eqs. 1, 2, and 3). A linear fit model without an offset applied to such estimates will 


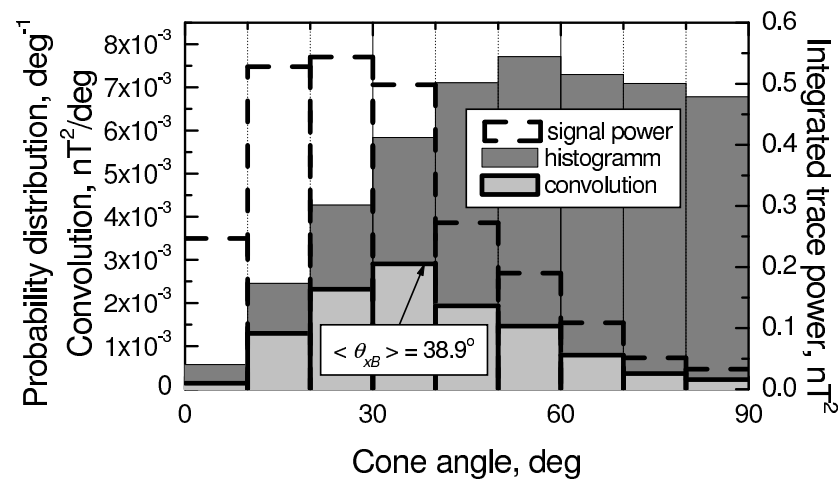

Fig. 12. Band-limited signal trace power vs $\theta_{x B}$ (dashed line), cone angle (dark shading), and their convolution (light shading). See comments in text.

cause the slope $C$ to be overestimated. It is necessary to mention here that earlier studies of the $f_{\max }\left(\left|B_{\mathrm{IMF}}\right|\right)$ dependence (Odera, 1986) also revealed a similar offset of $10-20 \mathrm{mHz}$, but these findings did not receive a satisfactory interpretation.

Finally, we should treat these results cautiously, taking into account all the assumptions and comments made in Sect. 3.2, i.e. the fixed slope $p=-4$ and the low $R$ values in the vicinity of $f_{\max }$.

\section{5 $\quad B_{z}$ and solar wind velocity}

The increase in broad band noise power for $B_{z}<0$ suggests that reconnection plays an important role in energy supply to the power-law component. However, it is still necessary to clarify whether this is connected with the noise generation mechanism, or with its propagation into the magnetosphere.

Figure 11 shows a steep increase in Pc3 signal, noise and Pc5 power with increasing solar wind velocity $V_{s w}$. Similar results have been reported in a number of previous studies of Pc3-4 at low, middle, and high latitudes on the ground and in space (Greenstadt et al., 1979a,b; Wolfe et al., 1980; Takahashi et al., 1981; Odera, 1986; Wolfe et al., 1987; Yumoto et al., 1987; Vellante et al., 1996; Morrison, 1991) and for Pc5 at cusp latitudes (Engebretson et al., 1998; Posch et al., 1999)). For example, Yedidia et al. (1991) performed a statistical analysis of daytime integrated Pc3 power recorded on the ground at low latitudes and found that the dependence of pulsation power on solar wind speed could be described by a power law of the form $\log P=-3.6+0.003 V_{s w}$. An increase in noise power with $V_{s w}$ at cusp latitudes was also reported by Engebretson et al. (1995) and Posch et al. (1999). However, it is important to note that, at these latitudes, the signal power grows faster than the noise, i.e. $R$ increases with increasing $V_{s w}$.

\section{Conclusions}

The results obtained in this work contribute to both magnetometer data processing and new experimental information about upstream Pc3 waves and broad band noise at cusp latitudes. In the past, it has not often been clear how researchers have processed their data, and these problem has been addressed. Our detailed analysis of average pulsation spectra from the cusp-latitude station Davis allowed us to determine a characteristic signature of band-limited Pc3-4 pulsations (signal) - a spectral enhancement between 10 and $100 \mathrm{mHz}$. These waves are likely produced in the upstream solar wind. In their absence, the background is characterized by a steep power-law spectrum with a slope $p \simeq-4$ (noise). This information was used to develop an algorithm for the effective separation of these two components, which was then applied to 3 years of cusp-latitude ground magnetometer data.

From our statistical analysis we found that the average signal-to-noise ratio at Davis exceeds unity between $\sim$ 09:00-14:00 MLT and over the $\sim 20-60 \mathrm{mHz}$ frequency range. Thus, band-limited Pc3 signals dominate the broad band power-law background for a few hours around local noon. Semiannual variations are present in both the signal and noise power components, with equinoctial maxima. The maximum signal power occurs at non-zero cone angles $\theta_{x B} \simeq 20^{\circ}$, while the noise power is almost invariant with cone angle. The signal frequency increases with increasing IMF magnitude as $f_{\max }[\mathrm{mHz}] \simeq 4.4\left|B_{\mathrm{IMF}}\right|[\mathrm{nT}]$. The signal power is maximum for $B_{z}=0$ (cone angle effect), while the noise power strongly favours $B_{z}<0$. The signal and noise power increase at different rates with increasing solar wind velocity. The variation of noise power with solar wind velocity is different for $B_{z}>0$ than for all $B_{z}$, while no such difference is seen for the band-limited Pc3 signal.

In interpreting these results, it is necessary to remember that different data processing, analysis, and visualization procedures applied to similar data sets may lead to different or even contradictory results or conclusions. This explains some of the discrepancies between our study and previous works, for example, in $\left|B_{\mathrm{IMF}}\right|$ or $R$ dependencies.

Our results clearly show that the Pc3-4 spectrum at cusp latitudes comprises significant contributions from physically different mechanisms that we have labelled signal and noise. The former is strongly connected with solar wind parameters and is most likely due to compressional mode waves generated by the ion-cyclotron instability upstream of the bow shock. This agrees with previous observations.

The source of the power-law noise is not clear. The diurnal variation is similar to that for Pc5 pulsations that are widely believed to be generated by the Kelvin-Helmholtz instability at the magnetopause. However, the noise power has a different dependence on solar wind cone angle and speed, and strongly favours $B_{z}<0$. This suggests the power-law noise generation mechanism is associated with reconnection. Future work needs to address this issue. For example, similar studies should extend to higher (polar cap) and lower (auroral region, plasmapause) magnetic latitudes, and 
the spectral deconvolution needs to be modified, to account for the observed spatial phase distribution and polarization structure of Pc3-4 waves.

Acknowledgements. This work was supported by the Australian Antarctic Division and the Antarctic Science Advisory Committee, the Australian Research Council, the University of Newcastle, and the Cooperative Research Center for Satellite Systems through the Commonwealth of Australia CRC Program. We are also grateful to the WIND MFI team from NASA/Goddard SFC who provided internet access to the WIND magnetometer data.

Topical Editor G. Chanteur thanks M. G. McHarg and K. Mursula for their help in evaluating this paper.

\section{References}

Bol'shakova, O. V. and Troitskaya, V. A.: The relation of the highlatitude maximum of Pc3 intensity to the dayside cusp, Geomagn. Aeron., 24, 633-635, 1984.

Cao, M., McPherron, R. L., and Russell, C. T.: Statistical study of ULF wave occurrence in the dayside magnetosphere, J. Geophys. Res., 99, 8731-8753, 1994.

Cliver, E. W., Kamide, Y., and Ling, A. G.: Mountains versus valleys: Semiannual variations of geomagnetic activity, J. Geophys. Res., 105, 2413-2424, 2000.

D'Angelo, N.: Ultralow frequency fluctuations at the polar cusp boundaries, J. Geophys. Res., 78, 1206-1209, 1973.

DeLauretis, M., Villante, U., and Vellante, M.: An analysis of power spectra indices in the micropulsation frequency range at different ground statons, Planet. Space Sci., 39, 975-982, 1991.

Engebretson, M. J., Meng, C. I., Arnoldy, R. L., and Cahill, L. J.: Pc3 pulsations observed near the south polar cap, J. Geophys. Res., 91, 8909-8918, 1986.

Engebretson, M. J., Anderson, B. J., Cahill, L. J., Arnoldy, R. L., Rosenberg, T. J., Carpenter, D. L., Gail, W. B., and Eather, R. H.: Ionospheric signatures of cusp latitude Pc3 pulsations, J. Geophys. Res., 95, 2447-2456, 1990.

Engebretson, M. J., Cahill, L. J., Arnoldy, R. L., Anderson, B. J., Rosenberg, T. J., Carpenter, D. L., Inan, U. S., and Eather, R. H.: The role of the ionosphere in coupling upstream ULF wave power into the dayside magnetosphere, J. Geophys. Res., 96, 1527-1542, 1991.

Engebretson, M. J., Hughes, W. J., Alford, L. J., Zesta, E., Cahill, L. J., Arnoldy, R. L., and Reeves, G. D.: Magnetometer array for cusp and cleft studies observations of the spatial extent of broadband ULF magnetic pulsations at cusp/cleft latitudes, J. Geophys. Res., 100, 19371-19386, 1995.

Engebretson, M. J., Glassmeier, K.-H., Stellmacher, M., Hughes, W. J., and Luhr, H.: The dependence of high-latitude Pc5 wave power on solar wind velocity and on phase of high-speed solar wind streams, J. Geophys. Res., 103, 26 271-26 283, 1998.

Engebretson, M. J., Cobian, R. K., Posch, J. L., and Arnoldy, R. L.: A conjugate study of Pc3-4 pulsations at cusp latitudes: Is there a clock angle effect?, J. Geophys. Res., 105, 15 965-15 980, 2000.

Fairfield, D. H.: Bow shock associated waves observed in the far upstream interplanetary medium, J. Geophys. Res., 74, 35413553, 1969.

Fraser-Smith, A. C.: ULF/lower-ELF electromagntic field measurements in the polar caps, Rev. Geophys. Space Phys., 20, 497$512,1982$.
Greenstadt, E. W., Olson, J. V., Loewen, P. D., Singer, H. J., and Russell, C. T.: Correlation of Pc3, 4, and 5 activity with solar wind speed, J. Geophys. Res., 84, 6694-6696, 1979a.

Greenstadt, E. W., Singer, H. J., Russell, C. T., and Olson, J. V.: IMF orientation, solar wind velocity, and Pc3-4 signals: a joint distribution, J. Geophys. Res., 84, 527-532, 1979b.

Gul'elmi, A. V., Plyasova-Bakunina, T. A., and Shchepetov, R. V.: Relations between the period of geomagnetic pulsations Pc3, 4 and the parameters of the interplanetary medium at the Earth's orbit, Geomagn. Aeron., 13, 331-, 1973.

Hoppe, M. M. and Russell, C. T.: Plasma rest frame frequencies and polarizations of the low-frequency upstream waves: ISEE 1 and 2 observations, J. Geophys. Res., 88, 2021-2028, 1983.

McHarg, M. G., Olson, J. V., and Newell, P. T.: ULF cusp pulsations: Diurnal variations and interplanetary magnetic field correlations with ground-based observations, J. Geophys. Res., 100, 19729-19742, 1995.

Menk, F. W., Fraser, B. J., Hansen, H. J., Newell, P. T., Meng, C.-I., and Morris, R. J.: Multistaion observations of Pc1-2 ULF pulsations in the vicinity of the polar cusp, J. Geomag. Geoelectr., 45, 1159-1173, 1993.

Morris, R. J. and Cole, K. D.: Pc3 manetic pulsations at Davis, Antarctica, Planet. Space Sci., 35, 1437-1447, 1987.

Morrison, K.: On the nature of Pc3 pulsations at $L=4$ and their solar wind dependence, Planet. Space Sci., 7, 1017-1023, 1991.

Odera, T.: Solar wind controlled pulsations: A review, Rev. Geophys., 24, 55-74, 1986.

Olson, J. V.: ULF signatures of the polar cusp, J. Geophys. Res., 91, 10 055-10 062, 1986.

Paschmann, G., Sckopke, N., Bame, S. J., Asbridge, J. R., Gosling, J. T., Russell, C. T., and Greenstadt, E. W.: Association of lowfrequency waves with suprathermal ions in the upstream solar wind, Geophys. Res. Lett., 6, 209-212, 1979.

Posch, J. L., Engebretson, M. J., Weatherwax, A. T., Detrick, D. L., Hughes, W. J., and Maclennan, C. G.: Characteristics of broadband ULF magnetic pulsations at conjugate cusp latitude stations, J. Geophys. Res., 104, 311-331, 1999.

Russell, C., Luhmann, J., Odera, T., and Stuart, W.: The rate of occurrence of dayside Pc3,4 pulsations: the L-value dependence of the IMF cone angle effect, Geophys. Res. Lett., 33, 10731079, 1985.

Samson, J. C.: Three-dimensional polarization characteristics of high-latitude Pc5 geomagnetic micropulsations, J. Geophys. Res., 77, 6145-6160, 1972.

Slawinski, R., Venkatesan, D., Wolfe, A., Lanzerotti, L. J., and Maclennan, C. G.: Transmission of solar wind hydromagnetic energy into the terrestrial magnetosphere, Geophys. Res. Lett., 15, 1275-1278, 1988.

Takahashi, K. and Anderson, B. J.: Distribution of ULF energy $(f<80 \mathrm{mHz})$ in the inner magnetosphere: a statistical analysis of AMPTE CCE magnetic field data, J. Geophys. Res., 97, 10751-10 773, 1992.

Takahashi, K., McPherron, R. L., Greenstadt, E. W., and Neeley, C. A.: Factors controlling the occurrence of Pc3 magnetic pulsations at synchronous orbit, J. Geophys. Res., 86, 5472-5484, 1981.

Takahashi, K., McPherron, R. L., and Terasava, T.: Dependence of the spectrum of Pc3-4 pulsations on the interplanetary magnetic field, J. Geophys. Res., 89, 2770-2780, 1984.

Vellante, M., Villante, U., Lauretis, M. D., and Barchi, G.: Solar cycle variation of the dominant frequencies of Pc3 geomagnetic pulsations at $L=1.6$, Geophys. Res. Lett., 23, 1505-1508, 
1996.

Wolfe, A., Lancerotti, L. J., and Maclennan, C. G.: Dependence of hydromagnetic energy on solar wind velocity and interplanetary magnetic field direction, J. Geophys. Res., 85, 114-118, 1980.

Wolfe, A., Kamen, E., Lanzerotti, L. J., Maclennan, C. G., Bamber, J., and Venkatesan, D.: ULF geomagnetic power at cusp latitudes in response to upstream solar wind conditions, J. Geophys. Res., 92, 168-174, 1987.

Wolfe, A., Kelman, R. D., Warren, S. E., Maclennan, C. G., and Lanzerotti, L. J.: Hydromagnetic frequency spectra in the high latitude quiet magnetosphere, Solar wind sources of magnetospheric ultra-low frequency waves, Geophysical monograph 81, AGU, Washington D.C., 375-385, 1994.
Yedidia, B. A., Vellante, M., Villante, U., and Lazarus, A. J.: A study of the relationship between micropulsations and solar wind properties, J. Geophys. Res., 96, 3465-3470, 1991.

Yumoto, K.: Low-frequency upstream wave as a probable source of low-latitude Pc3-4 magnetic pulsations, Planet. Space Sci., 33, 239-249, 1985.

Yumoto, K., Saito, T., Tsurutani, B. M., and Smith, E. J.: Relationship between the IMF magnitude and Pc3 magnetic pulsations in the magnetosphere, J. Geophys. Res., 89, 9731-9740, 1984.

Yumoto, K., Wolfe, A., Terasawa, T., Kamen, E., and Lanzerotti, L.: Dependence of Pc3 magnetic energy specra at South Pole on upstream solar wind parameters, J. Geophys. Res., 92, $12437-$ $12442,1987$. 